\title{
Separation of Variables and the Geometry of Jacobians ${ }^{\star}$
}

\author{
Jacques HURTUBISE
}

Department of Mathematics and Statistics, McGill University, 805 Sherbrooke St. W. Montreal H3A 2K6, Canada

E-mail: jacques.hurtubise@mcgill.ca

URL: http://www . math.mcgill.ca/hurtubise/

Received November 17, 2006, in final form January 08, 2007; Published online February 05, 2007

Original article is available at http://www.emis.de/journals/SIGMA/2007/017/

\begin{abstract}
This survey examines separation of variables for algebraically integrable Hamiltonian systems whose tori are Jacobians of Riemann surfaces. For these cases there is a natural class of systems which admit separations in a nice geometric sense. This class includes many of the well-known cases.
\end{abstract}

Key words: separation of variables; integrable Hamiltonian systems; geometry of Jacobians

2000 Mathematics Subject Classification: 37J35; 70H06

\section{Separation of variables and complex integrable systems}

The general subject of separation of variables is one with a long history and a wide variety of techniques, but, to a geometer at least, it is somewhat puzzling, if one is trying to find some geometric context which explains it. It does turn out, however, that from the viewpoint of complex geometry, there is a quite specific context in which there is some beautiful geometric content, and this is the subject of this survey. Over the years, I had the pleasure of discussing these questions several times with Vadim Kuznetsov, and always profited from his insights. It is an honour to be able to dedicate this article to his memory.

Separation of variables for real Hamiltonian integrable systems is, in some sense, automatic, at least if one is dealing with systems with compact level sets for the Hamiltonians and if one can reparametrize the ring of Hamiltonians: the existence of action-angle variables ensures that the Hamiltonian flows can be decoupled into one-dimensional problems. The level sets of the Hamiltonians are tori, and the angle coordinates express these as products of circles. The problem, of course, is not one of knowing that it can be done, but rather of doing it, in some fairly explicit way, on some explicit family of examples. Over the complex numbers, however, we shall see that the question of whether one can separate variables in some reasonable sense becomes tied in with the actual geometry of the level sets; the abstract existence of a separation becomes linked to its effective computation.

One can imagine starting with some real analytic integrable system, and complexifying it. A first question is whether the level sets of the real system, which we will assume to be compact and so, generically, tori, remain so once complexified, compactified, and eventually desingularised. This is far from being automatic; the passage to the complex domain reveals more elaborate geometry: one need only think of algebraic curves in the plane, which are unions of ovals; their complexifications reveal much more intricate structure. Nevertheless, there are real integrable systems which complexify well, in the sense that the level sets of the Hamiltonians are

\footnotetext{
*This paper is a contribution to the Vadim Kuznetsov Memorial Issue "Integrable Systems and Related Topics". The full collection is available at http://www.emis.de/journals/SIGMA/kuznetsov.html
} 
complex tori, that is, real tori topologically, but with a complex structure; we refer the reader to the paper [7] and the references therein for a discussion of this question of whether one still has tori over the complex domain, which is in fact linked, in the case when the tori are in addition algebraic (Abelian varieties), to the existence of large families of meromorphic local solutions, i.e., to some sort of Painlevé test.

We will assume that our integrable system, now complex of dimension $2 r$, has as Lagrangian leaves (level sets of the Hamiltonians) a family of open dense subsets of complex tori of complex dimension $r$, at least over some dense open set: the leaves compactify to give complex tori. In the examples, our tori are algebraic, and the systems are what is known as algebraically integrable.

Over the complex numbers, the tori are far from having a constant structure. One can, as in the real case, obtain action-angle variables $I_{j}, \theta_{j}$, so that the symplectic form is given by $\sum_{j} d I_{j} \wedge d \theta_{j}$ and the Lagrangian leaves $\mathcal{L}_{c}, c=\left(c_{1}, \ldots, c_{r}\right) \in \mathbb{C}^{r}$ are cut out by $I_{j}=c_{j}$. The tori are of the form

$$
\mathcal{L}_{c}=\mathbb{C}^{r} /\left(\mathbb{Z}^{r}+\Lambda(c) \cdot \mathbb{Z}^{r}\right),
$$

where $\Lambda(I)$ is some matrix-valued function of the $I_{j}$ with non-degenerate imaginary part. One can show that for there to be a symplectic form on the fibration of tori such that the individual tori are Lagrangian, one needs

$$
\frac{\partial \Lambda_{i j}}{\partial I_{k}}=\frac{\partial \Lambda_{i k}}{\partial I_{j}}
$$

so that $\Lambda_{i j}=\partial G_{i} / \partial I_{j}$ for some $G_{i}(I)$. This is the explicit version of the 'cubic condition' of Donagi-Markman [13]. It is simply a consequence of the need for the form $\sum_{j} d I_{j} \wedge d \theta_{j}$ to be invariant under translation by the lattice $\Lambda(I) \cdot \mathbb{Z}^{r}$.

Thus the functions $G_{i}(I)$ distinguish algebraically integrable systems, essentially by their derivatives being parameters for moduli of the tori in the fibers; one can derive invariants from them. We are interested in cases for which the tori are in some reasonable sense symmetric products of one dimensional compact manifolds, as this will be necessary for separation of variables in the integrable system.

A natural class for which this occurs are the Jacobians $J(S)$ of compact Riemann surfaces $S$. Indeed, if $S$ is such a surface, of genus $g$, one has a $g$-dimensional space of holomorphic one-forms on $S$; let $\omega_{i}, i=1, \ldots, g$ be a basis for this space. The integral of any one-form along $S$ from one point to another is only defined modulo periods. The first homology of $S$ is $\mathbb{Z}^{2 g}$; let $c_{j}$ be a (standard, symplectic) basis for this group. One can normalise so that the periods $\oint_{c_{j}} \omega_{i}=\delta_{i j}$, $i, j \in\{1, \ldots, g\}$; let $\Lambda_{i j}=\oint_{c_{g+j}} \omega_{i}$ generate the remaining periods. We set

$$
J(S)=\mathbb{C}^{g} /\left(\mathbb{Z}^{g}+\Lambda \cdot \mathbb{Z}^{g}\right) .
$$

Choosing a base point $p_{0}$, one has the Abel-Jacobi map

$$
A: S \rightarrow J(S), \quad p \mapsto \int_{p_{0}}^{p} \omega_{1}, \ldots, \omega_{g} .
$$

One can extend this map to the symmetric products of $S$ :

$$
A^{(r)}: S^{r} \rightarrow J(S), \quad\left(p_{1}, \ldots, p_{r}\right) \mapsto \sum_{i=1}^{r} \int_{p_{0}}^{p_{i}} \omega_{1}, \ldots, \omega_{g}
$$

The basic fact that drives a lot of what follows is the fact, due to Jacobi, that the map

$$
A^{(g)}: S^{g} \rightarrow J(S)
$$

is onto and generically bijective. 
In the case when the Lagrangian tori of the integrable systems are Jacobians, then, we are half way there in terms of having a separation of variables, in that we are decomposing the Jacobian as a symmetric product of the curve. The purpose of this note is to highlight some results concerning algebraically integrable systems for which a separation of variables of the whole phase space in terms of symmetric products is natural, extending the geometry of the Jacobians; as we are dealing with phase spaces, the symmetric product is of a surface, which should contain the curves in our family. Two sets of $g$ points will belong to the same Lagrangian leaf if they belong to the same curve in the family. The separation in these coordinates follows easily. The cases covered include many of the frequently studied examples. In the sections which follow, we will give a survey, first of results concerning the local geometry of integrable systems of Jacobians, showing that if suitable conditions are met they can be related to symmetric products of surfaces, or more accurately to Hilbert schemes of points. After that we discuss some important cases, covering the various Gaudin models, the Sklyanin brackets and their generalisations, such as generalised Hitchin systems. We will also discuss relations to multi-Hamiltonian structures, and close with a discussion of what happens when one replaces Jacobians by Prym varieties.

What follows is a survey of results proven elsewhere, in collaboration with Malcolm Adams, John Harnad, Mounia Kjiri and Eyal Markman [2, 24, 28, 27, 30, 29]. In relation to the Gaudin model, a simple version also appears in [35]. Another place in which similar computations can be found is [21]. In computational form, some cases of the results presented here have a long history: one already has the result for the rational Gaudin model in [20], which appeared in 1919!

\section{The local geometry of systems of Jacobians}

Let $U$ be some $g$-dimensional variety; as we are talking about local geometry, the typical $U$ would be some small open ball in $\mathbb{C}^{g}$. Let

$$
\mathbb{J} \rightarrow U
$$

be an integrable system of Jacobians, that is a symplectic $2 g$ dimensional variety fibering over $U$, such that the fibers $J\left(S_{h}\right), h \in U$ are Lagrangian and are Jacobians $J\left(S_{h}\right)$ of genus $g$ Riemann surfaces $S_{h}$. There is an associated family of Riemann surfaces

$$
\mathbb{S} \rightarrow U
$$

with fibers $S_{h}$ over $h \in U$. If we choose base sections, we have a fiberwise Abel map

$$
A: \mathbb{S} \rightarrow \mathbb{J}
$$

which commutes with the projections to $U$. We note that the fiberwise Abel map (1) extends to the fiberwise symmetric product

$$
A^{(g)}: \mathbb{S}_{U}^{(g)} \rightarrow \mathbb{J}
$$

Extending the corresponding results for individual Jacobians, $A^{(g)}$ is onto and generically bijective.

We can pull back the symplectic form $\Omega$ from $\mathbb{J}$ to $\mathbb{S}$. One has that $A^{*} \Omega$ never vanishes, essentially because the Abel map is an immersion. Its square, however, can, and we say:

Definition 1. The system has rank two if for a suitable choice of base sections the pull-back of the symplectic form satisfies

$$
A^{*} \Omega \wedge A^{*} \Omega=0
$$


A classical theorem of Darboux tells us that there are then coordinates $x, y$ such that $A^{*} \Omega=d x \wedge d y$; locally the form is pulled back from a surface.

We digress for a moment to discuss Hilbert schemes. In general, these parametrise subvarieties of an algebraic variety, or more properly subschemes (which allows for degeneracy, multiplicity and so on). The case we need is associated to a smooth surface $Q$. We denote by $\operatorname{Hilb}^{g}(Q)$ the Hilbert scheme which parametrises 0-dimensional schemes $D$ of $Q$ of length $g$. These schemes $D$ are generically unions of $g$ unordered points of $Q$, with more complicated structures when points coalesce. The space $\operatorname{Hilb}^{g}(Q)$ has a support map $\operatorname{Hilb}^{g}(Q) \rightarrow Q^{(g)}$ to the $g$-th symmetric product, which is an isomorphism over the set of $g$-tuples of distinct points. Hilb ${ }^{g}(Q)$ is smooth, and is a desingularisation of $Q^{(g)}$. When $Q$ is symplectic, or Poisson, $\operatorname{Hilb}^{g}(Q)$ is also symplectic, or Poisson, and the structure is a desingularisation of the natural one on $Q^{(g)}$. We note that more properly we should be talking of Douady spaces, as the surfaces we have are not apriori algebraic. Nevertheless, the local structure is identical to that of the well studied Hilbert schemes of points on a smooth algebraic surface, and we will keep the notation $\operatorname{Hilb}^{g}(Q)$.

Theorem 1 ([27]). (i) Let the system be of rank 2 . Under the embedding $A$, the variety $\mathbb{S}$ is coisotropic. Quotienting by the null foliation, one obtains, restricting $U$ if necessary, a surface $Q$ and a projection $\pi: \mathbb{S} \rightarrow Q$, such that $A^{*} \Omega$ projects, defining a symplectic form $\omega$ on $Q$ with $\pi^{*}(\omega)=A^{*}(\Omega)$. The Riemann surfaces $S_{h}$ all embed in $Q$.

(ii) (independence of base section) If $A, \tilde{A}$ are two Abel maps with $A^{*} \Omega \wedge A^{*} \Omega=0, \tilde{A}^{*} \Omega \wedge$ $\tilde{A}^{*} \Omega=0$, then $A^{*} \Omega=\tilde{A}^{*} \Omega$, when $g \geq 3$, and so $Q$ depends only on $\mathbb{S}$ and not on the particular Abel map chosen. For $g=2, A^{*} \Omega \wedge A^{*} \Omega$ is always zero.

(iii) There is a diagram of maps:

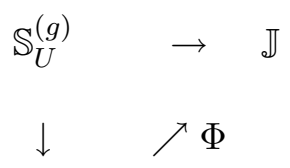

$\operatorname{Hilb}^{g}(Q)$

which defines the birational symplectic morphism $\Phi$, an isomorphism over Zariski open sets, between $\mathbb{J}$ and $\operatorname{Hilb}^{g}(Q)$. The image of symmetric products $S_{h}^{(g)}$ of the curves is Lagrangian in $\operatorname{Hilb}^{g}(Q)$, and the restriction of $\Phi$ to $S_{h}^{(g)}$ is the Abel map

$$
S_{h}^{(g)} \rightarrow J\left(S_{h}\right) .
$$

What this tells us, roughly, is that the surface $Q$ and the $g$-dimensional family of curves $S_{h}$, $h \in U$ embedded in it encodes the integrable system. The inclusion of the curve $S_{h}$ in $Q$ is for dimensional reasons Lagrangian, and the induced inclusion of symmetric products

$$
S_{h}^{(g)} \rightarrow Q^{(g)},
$$

which lifts to the Hilbert scheme,

$$
S_{h}^{(g)} \rightarrow \operatorname{Hilb}^{g}(Q),
$$

is still Lagrangian, and defines the Lagrangian leaves of the integrable system. The isomorphism $\Phi$ of Theorem 1 is, in effect, the change of variables to separating coordinates.

The key constraint, of course, is the rank 2 condition. A related construction, due to Krichever and Phong [32] uses the existence of a meromorphic one-form, whose exterior derivative is the symplectic form. 


\section{$3 \quad r$-matrix systems and their generalisations}

We now outline how a large family of well-studied integrable systems satisfy the rank 2 condition and therefore admit algebraic separation of variables. The list of systems comprises all of those defined using loop algebra-valued $r$-matrices, for the loop algebra $g l(r)$ :

- the rational $g l(r)$-Gaudin model and its degenerations [2, 23];

- the rational $G l(r)$ Sklyanin brackets (magnetic chain) [38, 40, 41];

- the elliptic and trigonometric $g l(r)$ Gaudin models [28];

- the elliptic and trigonometric $G l(r)$ Sklyanin brackets [30];

- the $G l(r)$-cases of the systems defined using the 'dynamical $r$-matrix' formalism of Felder, Etingof-Varchenko [14, 15, 17, 31];

- the $g l(r)$ Hitchin systems, and their generalisations [9, 25, 26, 27, 28, 33].

These systems contain many, if not most, of the examples of integrable systems of Jacobians that have been studied (see, for example, $[1,3,5,6,12,36,37]$ ). Their generalisations to structure groups other than $G l(r)$ are also very important, for example with the orthogonal groups in the study of tops; the Abelian varieties which occur naturally are not Jacobians, however, but a more general class known as Prym varieties. We shall discuss these briefly later.

We first outline how these systems are indeed integrable systems of Jacobians. Their phase spaces are all, (after quotienting out, in some cases, by groups of global automorphisms) spaces of pairs $(E, \phi)$, where $E$ is a vector bundle over a Riemann surface $\Sigma$ and $\phi$ is a meromorphic 1-form-valued section of the endomorphism bundle $\operatorname{End}(E)$ with poles over a positive divisor $D$ :

$$
\phi \in H^{0}\left(\Sigma, \operatorname{End}(E) \otimes K_{\Sigma}(D)\right) .
$$

For example, for the (reduced) rational Gaudin model, the Riemann surface is the projective line, the bundle $E$ is trivial, and so $\phi$ becomes a matrix valued meromorphic function with poles at $D$, considered modulo conjugation by a constant matrix.

Now let $X$ be the total space of the line bundle $K(D)$ over $\Sigma$ of one-forms with poles at $D$. One has a projection $\pi: X \rightarrow \Sigma$. To each pair $(E, \phi)$ one can associate a pair $(S, L)$. Here $S$ is the spectral curve of $\phi$, embedded in $X$, where it is cut out by the equation

$$
\operatorname{det}(\phi-\xi \mathbb{I})=0
$$

where $\xi$ is the tautological section of $\pi^{*} K(D)$ over $X$. L, in turn, is the sheaf defined over $X$ but supported over $S$ given as the cokernel of $\phi$ :

$$
0 \rightarrow \pi^{*} E \otimes \pi^{*} K^{*}(-D) \stackrel{\phi-\xi \mathbb{I}}{\longrightarrow} \pi^{*} E \rightarrow L \rightarrow 0
$$

One can recover the pair $(E, \phi)$ from $(S, L)$ as direct images, $E$ as the direct image of $L$, and $\phi$ as the direct image of multiplication by the tautological section:

$$
\begin{aligned}
& E=\pi_{*}(L), \\
& \phi=\pi_{*}(\times \xi) .
\end{aligned}
$$

One has that over the generic locus of smooth $S$, the sheaf $L$ is a line bundle over $S$, and is represented by a point in the Jacobian of $S$.

The nice thing is the map which associates to $(S, L)$ the pair $(E, \phi)$ is Poisson. Indeed, the surface $X$ has a Poisson structure(indeed, frequently, several), and it is the discovery of Mukai, Bottacin and Tyurin $[10,34,42]$ that this structure induces a Poisson structure on the variety 
of pairs $(S, L)$. The family of curves $S$ is a linear system, and is such that deformations of the curve which fix the intersection with the degeneracy divisor $D$ of the Poisson structure are given infinitesimally by sections of the canonical bundle of the curve; the line bundles are of fixed degree, and their deformations are given by the first cohomology of the structure sheaf of the curves. The Poisson structure is then derived from the Serre pairing of these two deformation spaces. We have, in all the cases quoted above (precise statements for the individual cases can be found in $[2,24,28,27,30,29])$ :

Proposition 1. For a suitable choice of Poisson structure on $X$, the map

$$
(S, L) \mapsto(E, \phi)
$$

is Poisson.

Viewed invariantly, the result is not difficult, and just expresses an invariance under 'pushdown'. See [24].

The integrable systems under study all have as Hamiltonians the coefficients of the spectral curve (2). One then shows:

Proposition 2. The fibration

$$
(S, L) \mapsto S
$$

is Lagrangian, with fiber over $S$ the Jacobian of $S$ when $S$ is smooth.

Finally, by tensoring $L$ by a fixed line bundle if necessary (which is always possible locally), we can suppose that the degree of $L$ is the genus $g$ of $S$. Generically, then, $L$ has only one section $s_{L}$ up to scale. Let $D_{L}$ be its divisor on $S . D_{L}$ has degree $g$, and is, generically, the union of $g$ distinct points of $S \subset X$. In any case, the divisor $D_{L}$ is cut out on $X$ by the equation of the spectral curve, and some additional equations, and so can be thought of as an element of Hilb $^{g}(X)$. A fairly straightforward computation shows that over the set where $L$ has a unique section up to scale:

Proposition 3. The map

$$
(S, L) \mapsto D_{L}
$$

is Poisson.

This, in essence, shows us that $X$ should be the variety $Q$ of the main theorem of the preceding section. This is not quite the case; $X$ is only Poisson, while $Q$ is symplectic. One finds, however, that the symplectic leaves of the original space of pairs $(E, \phi)$ give curves which intersect the degeneracy locus $V$ of the Poisson structure on $X$ at fixed points. Blowing up $X$ at these points gives our variety $Q$.

It should be emphasised that this correspondence between $(E, \phi)$ and a set of points on $X$, while presented here in an abstract way, is actually fairly effective computationally, so that if the original phase space can be described effectively, then so can the passage to $g$ points on $X$, and then the integration of the system. We illustrate this with one example, following from [30]; the general procedure is the same in all cases.

The example in question is that of the $G l(r, \mathbb{C})$ elliptic Sklyanin or quadratic $r$-matrix bracket. Let $\Sigma$ be an elliptic curve,

$$
\Sigma=\mathbb{C} /\left(\omega_{1} \mathbb{Z}+\omega_{2} \mathbb{Z}\right)
$$


and let $\rho: \mathbb{C} \rightarrow \Sigma$ be the natural projection. Let $q=\exp (2 \pi i / r)$, and define two $r \times r$ matrices

$$
I_{1}=\operatorname{diag}\left(1, q, q^{2}, \ldots, q^{r-1}\right), \quad I_{2}=\left(\begin{array}{ccccc}
0 & 1 & 0 & \cdots & 0 \\
0 & 0 & 1 & \cdots & 0 \\
\cdot & \cdot & \cdot & & \cdot \\
. & \cdot & \cdot & & . \\
0 & 0 & 0 & \cdots & 1 \\
1 & 0 & 0 & \cdots & 0
\end{array}\right) .
$$

Let $D$ be a positive divisor on $\Sigma$ :

$$
D=\sum_{i=1}^{n} \nu_{i}, \quad \nu_{i} \in \Sigma .
$$

(Repetitions are allowed among the $\nu_{i}$.) We set $\tilde{D}$ to be the lift of this divisor to $\mathbb{C}$, under the projection $\rho$; this is an infinite, but locally finite, sum of points. Our phase space $M^{D}$ will be the product of the curve with the space of meromorphic functions on $\mathbb{C}$ with values in $G l(r, \mathbb{C})$, having poles bounded by $\tilde{D}$ and satisfying some quasiperiodicity relations:

$$
\begin{aligned}
M^{D}= & \Sigma \times\{\phi \mid \phi \text { is a meromorphic } G l(r, \mathbb{C}) \text { - valued function on } \mathbb{C} \text { such that } \\
& \text { polar divisor } \left.(\phi) \leq \tilde{D} \text { and } \phi\left(\lambda+\omega_{i}\right)=I_{i} \phi(\lambda) I_{i}^{-1}, i=1,2\right\} .
\end{aligned}
$$

This phase space comes equipped with a quadratic bracket defined in terms of an elliptic $r$-matrix $[40,41]$. The Hamiltonians, as mentioned above, are the coefficients for the equation of the spectral curve of $\phi$.

The phase space $M^{D}$ is of the type we have been considering, though this is not evident at first sight. The key is a way of defining vector bundles over curves which goes back to Weil. In essence, if one has a flat degree zero bundle over the curve, sections of this bundle are given by sections of the trivial bundle over the universal cover satisfying appropriate automorphy relations, given by the holonomy matrices of the connection, which then form a representation of the fundamental group. In the case of an elliptic curve, the representation would be given by two commuting matrices. This is not the case here, as the commutator of $I_{1}, I_{2}$ is a root of unity. However, a generalisation of Weil's procedure, given for example in [22], allows one to construct bundles of non-zero degree; one takes representations not of the fundamental group but of a central extension of the fundamental group by appropriate roots of unity. For a degree one bundle, the relation required is the one satisfied by $I_{1}, I_{2}$. The bundle obtained in this way is stable. By a theorem of Atiyah [4], all stable bundles on the elliptic curve $\Sigma$ of degree one, rank $r$ are obtained from this one by tensoring by a line bundle $V$ of degree zero, so that the moduli space of stable bundles of degree one, rank $r$ is in fact parametrised by the line bundles (mod, in fact, the $r$-th roots of unity) and the parameter space is simply $J(\Sigma)=\Sigma$.

We have defined then a bundle $E$ whose sections are given over $\mathbb{C}$ as vector valued functions $s$ satisfying the automorphy relations:

$$
s\left(z+\omega_{i}\right)=I_{i} s(z) .
$$

This is not possible if one asks for the functions to be holomorphic on all of $\mathbb{C}$. One must allow some singularities; one chooses a point $p$ in $\Sigma$, and considers all of its inverse images $\tilde{p}$ in $\mathbb{C}$; holomorphic sections of $E$ correspond to functions $s$ of the form $z^{-1 / r}$.(holomorphic) near the points $\tilde{p}$, and otherwise holomorphic on the plane; here $z$ is some coordinate with $z=0$ corresponding to a $\tilde{p})$. In the same vein, sections of $\operatorname{End}(E)$ over $\Sigma$ correspond to holomorphic matrix valued functions over $\mathbb{C}$ satisfying the quasi periodicity conditions of (3). (For the 
endomorphisms, holomorphic sections now correspond to holomorphic functions.) The second factor of $(3)$ is thus the space $H^{0}(\Sigma, \operatorname{End}(E)(D))=H^{0}\left(\Sigma, \operatorname{End}(E) \otimes K_{\Sigma}(D)\right)$ of meromorphic sections with poles at $D$. (Recall that the bundle of one-forms is trivial on an elliptic curve.) As tensoring $E$ by a line bundle leaves the automorphism bundle unchanged, one has that the space $M^{D}$ is a moduli space of pairs $(E \otimes V, \phi)$ :

$$
M^{D}=\left\{\left(E^{\prime}, \phi\right) \mid E^{\prime} \text { stable, rank } r \text {, degree one over } \Sigma, \phi \in H^{0}(\Sigma, \operatorname{End}(E)(D))\right\} .
$$

$A$ basic section. One wants to extract from a pair $\left(E^{\prime}=E \otimes V, \phi\right)$ a divisor $D_{L}$. We recall that $L$ was defined as the cokernel of the map

$$
\phi-\xi \mathbb{I}: E \otimes V(-D) \rightarrow E \otimes V .
$$

One wants the divisor defined by the vanishing of a suitable section of $L$.

Sections $s=s(z)$ of $E \otimes V$ map to sections of $L$, by the map (4), and as sections of $L$ they vanish when as sections of $E \otimes V$ they lie in $(\phi-\xi \mathbb{I})(E \otimes V(-D))$. Generically, lying in the image of $(\phi-\xi \mathbb{I})$ is the same as lying in the kernel of the matrix of cofactors:

$$
(\phi-\xi \mathbb{I})_{\mathrm{adj}} \cdot s=0 .
$$

This equation will be satisfied at $g$ points $\left(z_{\mu}, \xi_{\mu}\right)$, and it is these points we want in terms of $\phi$. For this, we simply need to compute $s$.

In fact, it suffices to compute when $V$ is the trivial bundle. The reason is that translation $T_{z_{0}}$ by $z_{0}$ along the curve has the same effect on $E$ as tensoring by a line bundle, so that if $\left(z_{\mu}, \xi_{\mu}\right)$ are coordinates for $(E, \phi)$, then $\left(z_{\mu}-z_{0}, \xi_{\mu}\right)$ are coordinates for $\left(T_{z_{0}}^{*}(E), \phi\right)$. A consequence of this, by the way, is that taking the reduction of $(3)$ to $S l(r)$ is equivalent to dropping the first factor and asking that the matrices in the second factor belong to $S l(r, \mathbb{C})$. The coordinates $\left(z_{\mu}, \xi_{\mu}\right)$ then get shifted to their 'centre of mass':

$$
\left(z_{\mu}, \xi_{\mu}\right) \mapsto\left(z_{\mu}-g^{-1} \sum_{\nu} z_{\nu}, \frac{\xi_{\mu}}{\prod_{\nu} \xi_{\nu}}\right) .
$$

Returning to $G l(r)$, to compute we need a section $s(z)=\left(s_{1}(z), s_{2}(z), \ldots, s_{r}(z)\right)$ of $E$, expressed explicitly. This can be done as follows, following [28]. We normalise the periods of the elliptic curve $\Sigma$ to $\omega_{1}=1 / r, \omega_{2}=\tau / r$. Let us take the $r$-th powers $f_{i}=s_{i-1}^{r}$ of the components of $s$, so that

$$
s_{i}=f_{i+1}^{1 / r} .
$$

Let us 'puncture' the curve $\Sigma$ at the point $((1+\tau) / 2 r)$, and puncture the plane at the inverse images $((1+\tau) / 2 r)+(1 / r) \mathbb{Z}+(\tau / r) \mathbb{Z}$ of this point. We would then like to find an $r$-tuple $F$ of functions $\left(f_{0}, \ldots, f_{r-1}\right)$, which are of the form $\zeta^{-1}$ (holomorphic) ${ }^{r}$ near the punctures (where $\zeta$ is a coordinate centered on the puncture), satisfy

$$
F\left(z+\frac{1}{r}\right)=F(z), \quad F\left(z+\frac{\tau}{r}\right)=I_{2} \cdot F(z),
$$

and are such that the $r$-th roots along the real and imaginary axes satisfy

$$
\left(f_{i}\right)^{1 / r}\left(z+\frac{1}{r}\right)=q^{i}\left(f_{i}\right)^{1 / r}(z) \quad\left(f_{i}\right)^{1 / r}\left(z+\frac{\tau}{r}\right)=\left(f_{i+1}\right)^{1 / r}(z) .
$$

Since $I_{1}^{r}=I_{2}^{r}=1$, one is dealing with functions over the elliptic curve $\Sigma^{\prime}=\mathbb{C} /(\mathbb{Z} \oplus \tau \mathbb{Z})$; let $\theta$ be the standard theta function for this curve; recall that it has a simple zero at the points $((1+\tau) / 2)+\mathbb{Z}+\tau \mathbb{Z}$, and is otherwise non-zero and holomorphic. We distinguish two cases: 
Case 1: $r$ is odd.

Let

$$
\theta_{k, j}(z)=\theta\left(z+\frac{k+j \tau}{r}\right), \quad 0 \leq k, j \leq(r-1) .
$$

We have the relations:

$$
\begin{aligned}
& \theta_{k, j}(z+m)=\theta_{k, j}(z), \quad \theta_{k, j}(z+m \tau)=\exp \left(-\pi i m^{2} \tau-2 \pi i m\left(z+\frac{k+j \tau}{r}\right)\right) \theta_{k, j}(z), \\
& \theta_{k, j}\left(z+\frac{1}{r}\right)=\theta_{k+1, j}(z), \quad \theta_{k, j}\left(z+\frac{\tau}{r}\right)=\theta_{k, j+1}(z), \quad 0 \leq j<(r-1), \\
& \theta_{k, r-1}\left(z+\frac{\tau}{r}\right)=\theta_{k, 0}(z) \exp \left(-\pi i \tau-2 \pi i\left(z+\frac{k}{r}\right)\right),
\end{aligned}
$$

where $m$ is an integer. Now if

$$
\rho_{j}=\frac{r-1}{2}-j
$$

we set

$$
f_{j}(z)=\exp \left(2 \pi i \tau\left(\frac{-j r(r-1)}{2}+\frac{(r-1) j(j+1)}{2}\right)\right) \prod_{k=0}^{r-1}\left(\frac{\theta_{k, j}^{r-2}(z) \theta_{k, j}\left(z+\rho_{j} \tau\right)}{\prod_{\ell=0, \ell \neq j}^{r-1} \theta_{k, \ell}(z)}\right)
$$

Using the relations given for the $\theta_{k, j}$, one checks that it has the correct form near the punctures, and that (5) holds. Now let $\tau$ be imaginary. Let us consider the involutions $f(z) \mapsto f(-z)$, $f(z) \mapsto \overline{f(\bar{z})}$. Both these involutions preserve the poles and zeros of $f_{0}$. From this, one has that $f_{0}$ must be even, as $f_{0}(0) \neq 0$. Using the second involution, one can then multiply $f_{0}$ by a constant $c$ so that $c f_{0}(0)$ is real. The function is then real on both imaginary and real axes, and has no zeros on the axes. From this, one has that (6) holds for $f_{0}$. From the relations (3), (6) follows for the other $f_{i}$. Deforming $\tau$, the same then must hold for arbitrary $\tau$.

Case 2: $r$ is even.

We then set

$$
\xi_{k, j}(z)=\theta\left(z+\frac{2 k-1+2 j \tau-\tau}{2 r}\right), \quad 0 \leq k, j \leq(r-1) .
$$

We have the relations:

$$
\begin{aligned}
& \xi_{k, j}(z+m)=\xi_{k, j}(z), \quad \xi_{k, j}(z+m \tau)=\exp \left(-\pi i m^{2} \tau-2 \pi i m\left(z+\frac{2 k-1+2 j \tau-\tau}{2 r}\right)\right) \xi_{k, j}(z), \\
& \xi_{k, j}\left(z+\frac{1}{r}\right)=\xi_{k+1, j}(z), \quad \xi_{k, j}\left(z+\frac{\tau}{r}\right)=\xi_{k, j+1}(z), \quad 0 \leq j<(r-1), \\
& \xi_{k, r-1}\left(z+\frac{\tau}{r}\right)=\xi_{k, 0}(z) \exp \left(-\pi i \tau-2 \pi i\left(z+\frac{2 k-1-\tau}{2 r}\right)\right)
\end{aligned}
$$

where $m$ is an integer. We then define

$$
\rho_{j}=\frac{r}{2}-j
$$


and set

$$
f_{j}(z)=(-1)^{j} \exp \left(2 \pi i \tau\left(\frac{-j(r-1)}{2}\right)\right) \prod_{k=0}^{r-1}\left(\frac{\xi_{k, j}^{r-1}(z) \xi_{k, j}\left(z+\rho_{j} \tau\right)}{\prod_{\ell=0}^{r-1} \xi_{k, \ell}(z)}\right) .
$$

Again, the $r$-th roots of the $f_{j}$ define our section.

Separating coordinates. As explained above, when $V$ is trivial, the separating coordinates on $M^{D}$ corresponding to an element $\phi$ are given by the solutions $\left(z_{\mu}, \xi_{\mu}\right)$ of

$$
(\phi(z)-\xi \mathbb{I})_{\operatorname{adj}} \cdot s=0 .
$$

For $V$ non trivial and represented by the translation $z_{0}$ on the curve, the coordinates are $\left(z_{\mu}-z_{0}, \xi_{\mu}\right)$.

The symplectic form. As outlined above, the symplectic form, expressed in the divisor coordinates, is inherited from the surface $X$; if $(z, \xi)$ are the coordinates defined as above on the surface, and our symplectic form on the surface is $f(z, \xi) d z \wedge d \xi$, then the symplectic form in terms of the divisor coordinates is $\sum_{\mu} f\left(z_{\mu}, \xi_{\mu}\right) d z_{\mu} \wedge d \xi_{\mu}$. In our case, $f(z, \xi)=1 / \xi$.

The separation of variables. The actual separation of variables in terms of our Hamiltonians is given by a simple generating function. Choose a basis $H_{1}, \ldots, H_{g}$ for the space of Hamiltonians;

one has in addition a certain number of Casimirs, which we label as $H_{g+1}, \ldots, H_{k}$. Fixing the $H_{i}$ fixes the spectral curve, and so determines $\xi$ as a function of $z$ :

$$
\xi=\xi\left(z, H_{1}, \ldots, H_{k}\right) .
$$

Choosing a base point on the spectral curve, we set

$$
F\left(z_{1}, \ldots, z_{g}, H_{1}, \ldots, H_{k}\right)=\sum_{\mu} \int_{z_{0}}^{z_{\mu}} \ln \left(\xi\left(z, H_{1}, \ldots, H_{k}\right)\right) d z .
$$

The linearising coordinates of the flows are given by

$$
Q_{i}=\frac{\partial F}{\partial H_{i}}=\sum_{\mu} \int_{z_{0}}^{z_{\mu}} \xi^{-1} \frac{\partial \xi}{\partial H_{i}} d z, \quad i=1, \ldots, g .
$$

One can show that these are sums of Abelian integrals.

\section{Starting from surfaces}

One can invert the procedure outlined in the preceding section, and start from a symplectic surface, or indeed a Poisson surface, and create an integrable system; these systems, in some sense, arrive already separated. This approach is in fact quite fruitful, and has been explained by Vanhaecke in two interesting papers $[43,44]$.

The ingredients in general are a Poisson surface $X$ (affine or projective) and a family of curves $S_{b}, b \in B$ on the surface. We ask that $B$ map surjectively to a variety $C$, whose functions will be the Casimirs of the construction. One then has an induced Poisson structure on the fiberwise $d$-th symmetric product over $B$ of the family of curves, where $d$ is the difference of dimensions between $B$ and $C$. The symplectic leaves are then the fibers over $C$. Already, the construction yields many interesting examples with $X=\mathbb{C}^{2}$.

One can restrict a bit more the geometry of the construction. Let $V$ be the divisor over which the Poisson tensor of the surface vanishes. Let us suppose that the surface is projective, that the 
system of curves has no base points, and let $C$ be the variety describing the intersection of the curves with $V$, so that the family of curves parametrised in $B$ as the inverse image of any given point in $C$ have fixed intersection in $X$ with $V$. One can then show that the fibers of $B$ over $C$ are $g$-dimensional, where $g$ is the genus of the generic curve of the family, and that the normal vector fields describing variations of curves within these fibers are the images under the Poisson tensor of holomorphic forms on the curves. When one goes to the ( $g$-dimensional) fiberwise symmetric product of the curves over $B$, one obtains a Poisson manifold which is birational to the family of Jacobians.

The family of projective Poisson surfaces is unfortunately a bit limited, mainly because such surfaces must have Kodaira dimension 0 or $-\infty$. Noting that Poisson structures map well under blowing down, one is reduced essentially to Abelian surfaces, K3 surfaces, (both symplectic), ruled surfaces and the projective plane. One can show that the ruled surface over a curve $\Sigma$, with a few exceptions, must be of the form $\mathbb{P}(\mathcal{O} \oplus \mathcal{K}(D))$, where $D$ is a positive divisor on the curve. These, in essence, are already covered by the cases outlined above, in Section 3.

\section{Comments}

\subsection{Multi-Hamiltonian structures}

The picture presented above, of decomposition into symmetric products, is also natural in the context of multi-Hamiltonian structures. The key point here is that any linear combination of Poisson structures in two dimensions is a Poisson structure. This principle propagates to symmetric products and gives a multi-Hamiltonian structure on the system. This gives one way of approaching multi-Hamiltonian structures examined in a certain number of recent papers on particular cases of the systems discussed above (e.g. [8, 18]).

For example, if one considers the space $M_{\mathrm{rat}, n}$ of matricial polynomials of degree at most $n$, one can build an $n+3$ dimensional space of Poisson structures on this space which incorporates both the rational linear $r$-matrix bracket and the rational quadratic (Sklyanin) bracket. These Poisson structures are induced in the way outlined in Section 3 from an $n+3$ dimensional family of Poisson structures on the total space of the line bundle $\mathcal{O}(n)$. In the tensor bracket notation of [19]:

$$
\left\{\phi(\lambda)^{\otimes}, \phi(\mu)\right\}_{a, b}:=\left[r(\lambda-\mu), \phi(\lambda) \otimes\left(a(\mu) \mathbb{I}-\frac{b}{2} \phi(\mu)\right)+\left(a(\lambda) \mathbb{I}-\frac{b}{2} \phi(\lambda)\right) \otimes \phi(\mu)\right] .
$$

Here $a(\lambda)$ is an arbitrary polynomial of degree at most $(n+1)$, and $b$ is an arbitrary constant.

The spectral curve procedure outlined above gives us a spectral curve in the total space $X$ of the line bundle $\mathcal{O}(n)$ over $\mathbb{P}^{1}$. The space $X$ has a family of Poisson structures given in standard coordinates by $(a(z)+b \xi)(\partial / \partial z \wedge \partial / \partial \xi)$, and a result of [24] tells us that these structures correspond to the family of brackets given above under the passage to divisor coordinates. In fact, the coordinates $\left(z_{\mu}, \xi_{\mu}\right)$ are Nijenhuis coordinates in a suitable sense for this family of Poisson structures [24].

The same holds also in the elliptic and trigonometric cases; again, see [24].

We should note that the existence of multi-Hamiltonian structures does not always imply that individual vector fields are multi-Hamiltonian, i.e., that for the field $V$ there are functions $f_{i}$ such that $V$ is generated by $f_{i}$ under the $i$ th Poisson structure. This question is explained in $[43,44]$.

\subsection{Other groups and Prym varieties}

As we noted above, integrable systems of the types listed above exist for complex reductive groups other than $G l(r)$. They do not quite fit into our set-up, as they are not systems of 
Jacobians, but rather of Prym varieties. Indeed, the construction we gave relied heavily on the existence of a spectral curve, that is a curve of eigenvalues of $\phi$. The analogous object in the general case, as developed in $[11,16,39]$ is the curve $C$ of conjugates of $\phi$ in the Lie algebra $\mathfrak{h}$ of a maximal torus $H$. This curve is Galois over the base curve, with Galois group the Weyl group of $G$. The analog of a line bundle in turn is a Weyl-invariant bundle $P_{H}$ with $H$ as structure group. Let $\mathcal{L}$ denote the integer lattice in $\mathfrak{h}$. The analogue of the Jacobian, classifying invariant $H$-bundles, is given by

$$
\left(J(C) \otimes_{Z} \mathcal{L}\right)^{W} .
$$

The connected component of the identity is known as a generalised Prym variety. The Lagrangian leaves of our systems are of this type.

There is for integrable systems of Prym varieties a corresponding notion of being of rank 2; one obtains, not a surface $Q$, but a variety $P$ of dimension $r+1$, where $r=\operatorname{dim}(H)$ [29]. This variety, and the family of curves, also encodes the system. The cases of the examples cited above, generalised to arbitrary $G$ are also of rank 2 in this generalised sense. There are no longer natural separating coordinates, but one can perform the actual integration to obtain variables $Q_{i}$.

Indeed, choosing a suitable basis $e_{i}$ for $\mathfrak{h}, H$-bundles decompose as a sum of line bundles $L_{i}$; one can for each of these obtain divisor functions $z_{i, \mu}, \xi_{\mu}^{i}$, where $\xi^{i}$ is the coordinate of the spectral curve (which lies in $K(D) \otimes \mathfrak{h}$ ) in the direction $e^{i}$ dual to $e_{i}$. These functions no longer form a coordinate system, as there is some redundancy due to the $W$-invariance. The symplectic form is still $\sum_{i, \mu} f\left(z_{i, \mu}, \xi_{\mu}^{i}\right) d z_{i, \mu} \wedge d \xi_{\mu}^{i}$, though, and writing $\xi$ as a function of $z$ and the independent Hamiltonians $H_{i}$ as above, defining the same generating function, as above, gives again the dual variables $Q_{i}$.

\section{References}

[1] Adams M.R., Harnad J., Hurtubise J., Isospectral Hamiltonian flows in finite and infinite dimensions. II. Integration of flows, Comm. Math. Phys. 134 (1990), 555-585.

[2] Adams M.R., Harnad J., Hurtubise J., Darboux coordinates and Liouville-Arnold integration in loop algebras, Comm. Math. Phys. 155 (1993), 385-413, hep-th/9210089.

[3] Adams M.R., Harnad J., Previato E., Isospectral Hamiltonian flows in finite and infinite dimensions. I. Generalised Moser systems and moment maps into loop algebras, Comm. Math. Phys. 117 (1988), 451-500.

[4] Atiyah M.F., Vector bundles over an elliptic curve, Proc. London Math. Soc. 7 (1957), 414-452.

[5] Adler M., van Moerbeke P., Completely integrable systems, Euclidean Lie algebras, and curves, Adv. Math. 38 (1980), 267-317.

[6] Adler M., van Moerbeke P., Linearization of Hamiltonian systems, Jacobi varieties and representation theory, Adv. Math. 38 (1980), 318-379.

[7] Adler M., van Moerbeke P., The complex geometry of the Kowalewski-Painlevé analysis, Invent. Math. 97 (1989), 1-46.

[8] Bartocci C., Falqui G., Pedroni M., A geometric approach to the separability of the Neumann-Rosochatius system, Differential Geom. Appl. 21 (2004), 349-360, nlin.SI/0307021.

[9] Bottacin F., Symplectic geometry on moduli spaces of stable pairs, Ann. Sci. École Norm. Sup. (4) 28 (1995), 391-433.

[10] Bottacin F., Poisson structures on moduli spaces of sheaves over Poisson surfaces, Invent. Math. 121 (1995), 421-436.

[11] Donagi R., Spectral covers, in Current Topics in Complex Algebraic Geometry (Berkeley, CA 1992/93), Math. Sci. Res. Inst. Publ. 28 (1995), 65-86, alg-geom/9505009.

[12] Donagi R., Markman E., Spectral curves, algebraically completely integrable Hamiltonian systems, and moduli of bundles, Lecture Notes in Math., Vol. 1620, Springer, Berlin, 1996, 1-119, alg-geom/9507017. 
[13] Donagi R., Markman E., Cubics, integrable systems, and Calabi-Yau threefolds, Israel Math. Conf. Proc. 9 (1996), 119-121, alg-geom/9408004.

[14] Etingof P., Schiffmann O., Twisted traces of intertwiners for Kac-Moody algebras and classical dynamical $R$-matrices corresponding to generalized Belavin-Drinfeld triples, Math. Res. Lett. 6 (1999), 593-612, math.QA/9908115.

[15] Etingof P., Varchenko A., Geometry and classification of solutions of the classical dynamical Yang-Baxter equation, Comm. Math. Phys. 192 (1998), 77-120, q-alg/9703040.

[16] Faltings G., Stable G-bundles and projective connections, J. Algebraic Geom. 2 (1993), 507-568.

[17] Felder G., Conformal field theory and integrable systems associated to elliptic curves, in Proceedings of the International Congress of Mathematicians (1994), Vol. 1, 2, Birkhäuser, Basel, 1995, 1247-1255, hep-th/9407154.

[18] Falqui G., Pedroni M., Separation of variables for bi-Hamiltonian systems, Math. Phys. Anal. Geom. 6 (2003), 139-179, nlin.SI/0204029.

[19] Faddeev L.D., Takhtajan L.A., Hamiltonian methods in the theory of solitons, Springer-Verlag, Berlin, 1987.

[20] Garnier R., Sur une classe de systèmes différentiels abéliens déduits de la théorie des équations linéaires, Rend. Circ. Mat. Palermo 43 (1919), 155-191.

[21] Gorsky A., Nekrasov N., Rubtsov V., Hilbert schemes, separated variables, and D-branes, Comm. Math. Phys. 222 (2001), 299-318, hep-th/9901089.

[22] Grothendieck A., Sur le mémoire de Weil: généralisations de fonctions abéliennes, Séminaire Bourbaki (1956-57) 9 (1959), Exposé no. 141.

[23] Harnad J., Hurtubise J., Generalised tops and moment maps into loop algebras, J. Math. Phys. 37 (1991), 1780-1787.

[24] Harnad J., Hurtubise J., Multi-Hamiltonian structures for r-matrix systems, CRM Preprint 2850, math-ph/0211076.

[25] Hitchin N.J., The self-duality equations on a Riemann surface, Proc. London Math. Soc. 55 (1987), 59-126.

[26] Hitchin N.J., Stable bundles and integrable systems, Duke Math. J. 54 (1987), 91-114.

[27] Hurtubise J., Integrable systems and algebraic surfaces, Duke Math. J. 83 (1996), 19-50.

[28] Hurtubise J., Kjiri M., Separating coordinates for the generalized Hitchin systems and the classical $r$-matrices, Comm. Math. Phys. 210 (2000), 521-540.

[29] Hurtubise J., Markman E., Rank 2 integrable systems of Prym varieties, Adv. Theor. Math. Phys. 2 (1998), 633-695, math.AG/9804059.

[30] Hurtubise J., Markman E., Surfaces and the Sklyanin bracket, Comm. Math. Phys. 230 (2002), 485-502, math.AG/0107010.

[31] Hurtubise J., Markman E., Elliptic Sklyanin integrable systems for arbitrary reductive groups, Adv. Theor. Math. Phys. 6 (2002), 873-978, math.AG/0203031.

[32] Krichever I.M., Phong D.H., On the integrable geometry of soliton equations and $N=2$ supersymmetric gauge theories, J. Differential Geom. 45 (1997), 349-389, hep-th/9604199.

[33] Markman E., Spectral curves and integrable systems, Compos. Math. 93 (1994), 255-290.

[34] Mukai S., Symplectic structure of the moduli space of sheaves on an abelian or K3 surface, Invent. Math. 77 (1984), 101-116.

[35] Novikov S.P., Veselov A.P., Poisson brackets and complex tori, Proc. Steklov Inst. Math. 165 (1984), $53-65$.

[36] Reiman A.G., Semenov-Tian-Shansky M.A., Reduction of Hamiltonian systems, affine Lie algebras and Lax equations I, II, Invent. Math. 54 (1979), 81-100, Invent. Math. 63 (1981), 423-432.

[37] Reiman A.G., Semenov-Tian-Shansky M.A., Integrable systems II, Chapter 2, in Dynamical Systems VII, Encyclopaedia Math. Sci., Vol. 16, Editors V.I. Arnold and S.P. Novikov, Springer-Verlag, Berlin, 1994.

[38] Scott D.R.D., Classical functional Bethe ansatz for $S L(N)$ : separation of variables for the magnetic chain, J. Math. Phys. 35 (1994), 5831-5843, hep-th/9403030.

[39] Scognamillo R., An elementary approach to the abelianization of the Hitchin system for arbitrary reductive groups, Compos. Math. 110 (1998), 17-37, alg-geom/9412020.

[40] Sklyanin E.K., On the complete integrability of the Landau-Lifschitz equation, LOMI Preprint E-3-79, 1979. 
[41] Sklyanin E.K., Poisson structure of a periodic classical XYZ-chain, J. Sov. Math. 46 (1989), $1664-1683$.

[42] Tyurin A., Symplectic structures on the varieties of moduli of vector bundles on algebraic surfaces with $p_{g}>0$, Math. USSR-Izv. 33 (1989), no. 1, 139-177.

[43] Vanhaecke P., Integrable Hamiltonian systems associated to families of curves and their bi-Hamiltonian structure, Progr. Math., Birkhäuser, "Systèmes intégrables et feuilletages", 1997, 187-212.

[44] Vanhaecke P., Integrable systems and symmetric products of curves, Math. Z. 227 (1998), 93-127, solv-int/9402002. 